\title{
Mid-term meeting of SELECTA: an European Training Network on smart electrodeposited alloys for environmentally sustainable applications
}

\author{
Uta Klement ${ }^{1}$, Eva Pellicer ${ }^{2}$, Jordi Sort ${ }^{2,3}$ \\ ${ }^{1}$ Department of Materials and Manufacturing Technology, Chalmers University of Technology, \\ Gothenburg 41296, Sweden \\ ${ }^{2}$ Departament de Física, Universitat Autònoma de Barcelona, 08193 Bellaterra, Spain \\ ${ }^{3}$ Institució Catalana de Recerca i Estudis Avançats (ICREA), Pg. Lluís Companys 23, 08010 Barcelona, Spain
}

e-mails: uta.klement@chalmers.se, eva.pellicer@uab.cat, jordi.sort@uab.cat

Integrating sustainability with technological progress is becoming one of the major challenges in modern society. Since research agendas are mainly driven by the push for incessant economic growth, there is the risk of reaching a critical point where technological advances will no longer be possible due to the imbalance between demand and availability of natural resources. To face this issue, the European Training Network "Smart ELECTrodeposited Alloys for environmentally sustainable applications: from advanced protective coatings to micro/nano-robotic platforms" (SELECTA) was launched in January 2015. SELECTA aims to integrate technological progress with environmental sustainability concerns, which is one of the major "Societal Challenges" listed in the Horizon 2020 Work Programme.

SELECTA is a joint academic-industry inter-disciplinary Network that focuses on the "green" electrochemical synthesis and characterization of metallic alloys, with tunable composition and microstructure, and its subsequent implementation in the following environmental / sustainable development applications: (a) advanced protective coatings; (b) robust and durable, energy-efficient micro/nano-electromechanical systems (MEMS/NEMS); (c) innovative micro/nano-robotic platforms for environmental healing. The project brings together 10 Beneficiaries and 6 Partner Organizations (including 5 private companies), belonging to 10 European Union Member States (plus Switzerland and Serbia).

The primary goal of SELECTA is to train 15 young researchers to pursue their PhDs in the field of smart electrodeposited metallic alloys suitable for environmental / sustainable development applications. The Network explores new types of electrodeposited alloys (essentially based on $\mathrm{Fe}, \mathrm{Cu}$ or $\mathrm{Al}$; free from hazardous and scarce raw elements), with 
tunable structure (amorphous, nanocrystalline), morphology (dense, nanoporous) and geometry (films, micropillars, nanowires), to meet specific technological demands (high wear/corrosion resistance, superior magnetic properties or hydrophobicity). Several disciplines (Physics, Electrochemistry, Engineering, Environmental Sciences, Biology and Robotics) converge together to provide a holistic approach to accomplish the SELECTA goals. World-class research is combined with unique training opportunities in soft skills, such as career planning, dissemination, intellectual property rights, entrepreneurship or management. The Network aims to provide highly-qualified specialists able to face future professional challenges in either Academia or Industry in an independent manner.

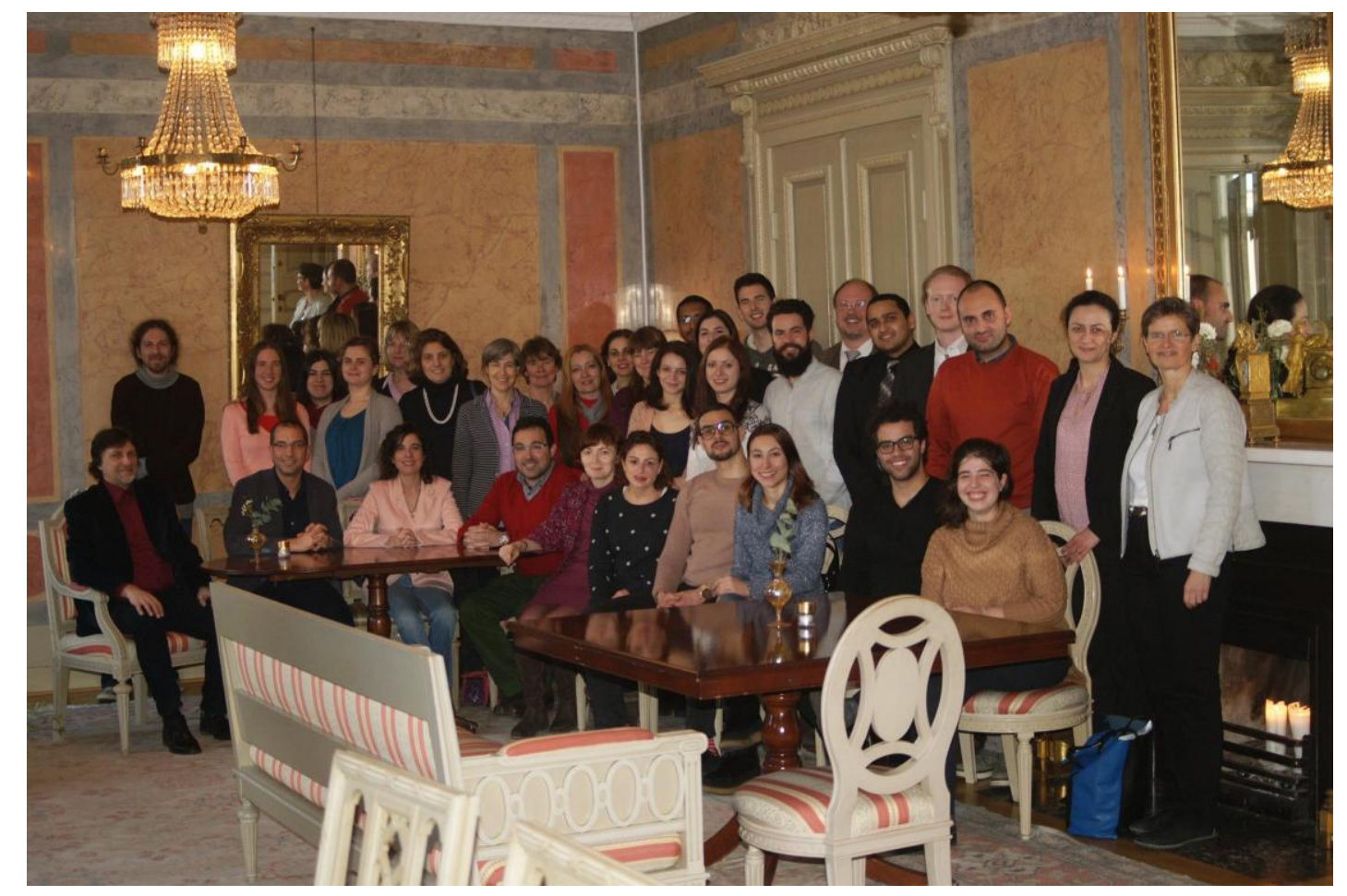

Participants at the SELECTA Mid-Term Review Meeting (Goteborg, Sweden, December 5-9 ${ }^{\text {th }} 2016$ )

Among the different activities organized within the SELECTA Network are a series of Workshops, a mid-term review meeting and Summer and Winter Schools. Distinguished researchers in fields related to electrodeposited materials are invited to deliver presentations to the members of the Network. Very recently, the SELECTA mid-term review meeting took place in Gothenburg (Sweden). Prof. Björn Johansson from Chalmers University of Technology delivered a talk on "Sustainability awareness for researchers", while Dr. Yiming Yao (also from Chalmers) explained the principles of the energy backscattered diffraction 
technique, as a means to perform texture analyses on electrodeposited films. In turn, Prof. Peter Leisner from SP Technical Research Institute of Sweden gave an excellent presentation entitled "What you also need to consider to make innovation out of promising research results". Further talks on sustainability and intellectual property rights and innovation aspects were delivered by Prof. K. Gårdfeldt (Head of Centre for Environment and Sustainability (GMV) at Chalmers and University of Gothenburg) and André Kelkkanen (from Chalmers Ventures), respectively. The whole event was warmly organized by Prof. Uta Klement.

SELECTA is arranged in 6 scientific Work-Packages (WP2-WP7), plus one Work-Package on management (WP1), as illustrated in the following diagram:

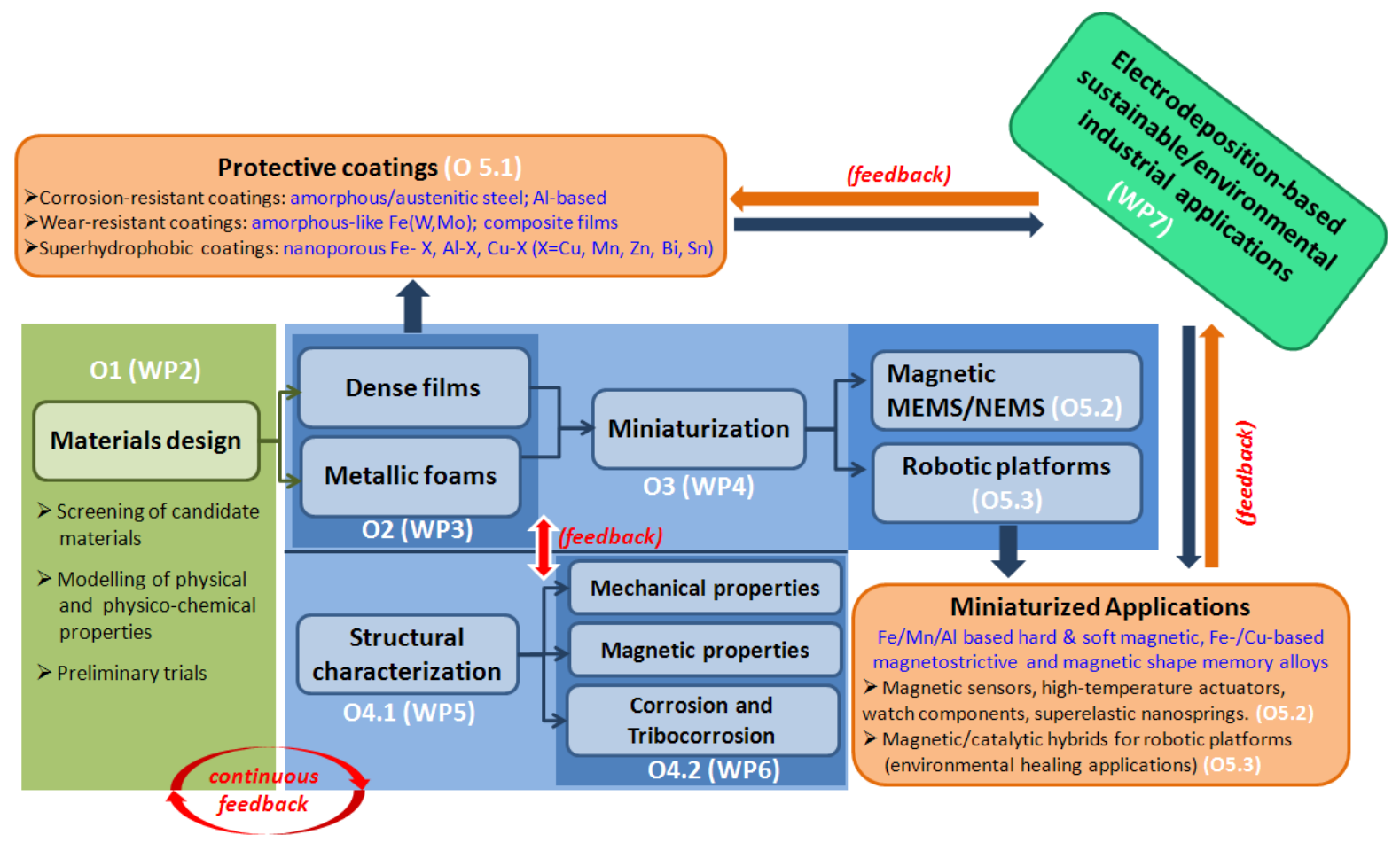

Illustration of the overall research structure of the SELECTA-ETN. Indicated are the objectives $(\mathrm{O})$ and the Work Packages (WP) of the research programme.

The WPs cover the most important aspects to develop and integrate materials by means of electrochemical processes in diverse applications, from protective coatings to miniaturized devices, under the umbrella of sustainability (WP7). Most of the objectives are fulfilled in a synergetic manner. This means that $\mathrm{PhD}$ students recruited under one of the WPs are integrated in the overall research programme by means of secondments to other Institutions, and through samples exchange, videoconferences, workshops and other planned activities. 
SELECTA aims to become a unique Research and Training platform that will generate a pool of highly skilled young researchers capable of designing, synthesizing, characterising and managing application-validated electrodeposited materials suitable for sustainable industrial activities. Collaborations with research institutions outside the Network are also possible. During the forthcoming two years, SELECTA aims to furnish Europe with a new generation of talented researchers with the necessary interdisciplinary knowledge to face the growing environmental/sustainability concerns of modern society in a proficient manner, filling the gaps between Materials Science and Sustainability/Environment, and between basic science and final market applications. Among the innovative research aspects of SELECTA, one can mention the following:

Replacement of cadmium and hard chromium in protective coatings remains an unresolved issue because the proposed alternative materials ( $\mathrm{CoP}, \mathrm{CoW}, \mathrm{NiP}, \ldots)$ contain large amounts of $\mathrm{Co}$ and $\mathrm{Ni}$, whose production is already being targeted as hazardous in most recent environmental legislations. The new target materials proposed in SELECTA are rather challenging from a synthetic point of view and the electrodeposition protocols still need to be clearly defined. SELECTA is tackling the fabrication of new alloys for corrosion-resistance applications (including in hightemperature and/or harsh environments).

Electrodeposition of magnetic alloys with outstanding magnetic properties, free from rare-earth free and noble-metals and containing neither Ni nor Co (or little amounts of both elements), is also not straightforward. Some of the proposed Fe-based compositions have been predicted to exhibit good magnetic properties but they have not been experimentally developed yet. New compositions need to be designed, particularly since $\mathrm{Fe}$ alloys tend to corrode rather easily. Corrosion studies in miniaturized systems are, on the other hand, scarce and are intended to be pursued within the Network.

Combining electrodeposition with miniaturization strategies at the nanoscale is also quite challenging and innovative. Difficulties arise either when using top-down approaches (laser machining with sub-micrometre precision is not easy) or bottom-up techniques (filling nanometre-sized pores in lithographed templates by means of electrodeposition is always not straightforward). Prototypes and industrial market studies of the following MEMS/NEMS are expected: ultra-sharp magnetic tips for 
MFM, watch components, high-temperature magnetic actuators or magnetic nanosprings/helices.

$>$ Implementation of magnetically-manoeuvred micro/nano-robotic platforms for environmental remediation applications also poses some challenges: it requires the use of complex shapes (for example, energy-efficient motion mimicking that of the E-coli bacteria involves the use of helical structures); perfect adherence between the magnetic and non-magnetic counterparts, as well as good mechanical integrity. New approaches are explored by SELECTA Team members.

From these lines, we would like to encourage interested Research Institutes, Universities or Companies to collaborate with the SELECTA Consortium. The Network will still continue during the next two years and the progress in the field requires of synergetic efforts by the scientific community.

SELECTA Coordinator: Prof. Jordi Sort (ES)

SELECTA Training Manager: Dr. Eva Pellicer (ES)

Mid-Term meeting Coordinator: Prof. Uta Klement (SE)

Participating Institutions: Universitat Autònoma de Barcelona, University of Cambrige, University of Ioannina, Chalmers University of Technology, Leibniz Institute for Solid Research and Materials Research Dresden, Vilnius University, Faculty of Engineering, University of Kragujevac, Istituto Nazionale di Ricerca Metrologica, Happy Plating GmbH, Swiss Federal Institute of Technology in Zurich, Eidgenoessische materialpruefungs- und forschungsanstalt, Electrodeposition of Stainless Steel Ltd., Aluinvent Zrt., IK4-CIDETEC Centre for Electrochemical Technologies, Yellow Research

More information of this European Training Network and related activities can be found at:

1. http://selecta-etn.eu/

Follow us in facebook: https://www.facebook.com/selecta2015/?fref=ts 